\title{
Two-Dimensional Black Phosphorus Carbide: Rippling and Formation of Nanotubes
}

\author{
Stepan A. Shcherbinin, Kun Zhou, Sergey V. Dmitriev, Elena A. Korznikova, Artur R. Davletshin, \\ and Andrey A. Kistanov*
}

Cite This: J. Phys. Chem. C 2020, 124, 10235-10243

Read Online

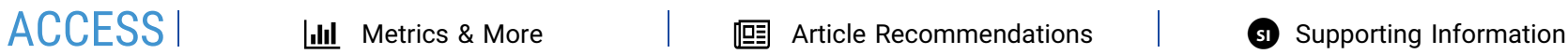

ABSTRACT: The allotropes of a new layered material, phosphorus carbide (PC), have been predicted recently, and a few of these predicted structures have already been successfully fabricated. Herein, by using first-principles calculations, we investigate the effects of rippling an $\alpha$-PC monolayer, one of the most stable modifications of layered PC, under large compressive strains. Similar to phosphorene, layered PC is found to have the extraordinary ability to bend and form ripples with large curvatures

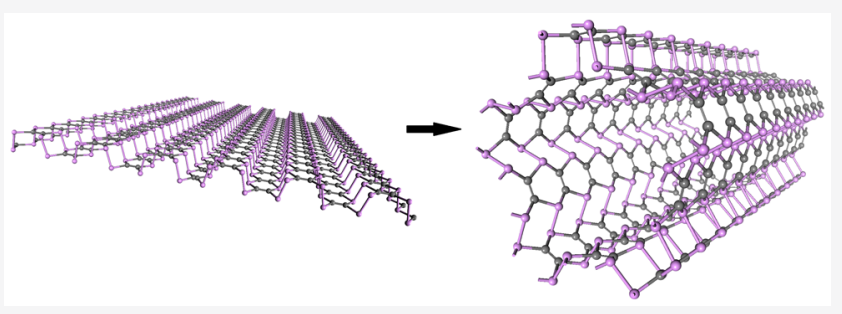
under a sufficiently large strain applied along its armchair direction.

The band gap, work function, and Young's modulus of a rippled $\alpha$-PC monolayer are predicted to be highly tunable by strain engineering. Moreover, a direct-indirect band gap transition is observed under compressive strains in the range from $6 \%$ to $11 \%$. Another important feature of the $\alpha$-PC monolayer rippled along the armchair direction is the possibility of its rolling to a PC nanotube (PCNT) under an extreme compressive strain. These tubes of different sizes exhibit high thermal stability, possess a comparably high Young's modulus, and a well tunable band gap which can vary from 0 to $0.95 \mathrm{eV}$. In addition, for both structures, rippled $\alpha$-PC and PCNTs, the changes of their properties under compressive strain are explained in terms of the modification of their structural parameters.

\section{INTRODUCTION}

Modern experimental techniques developed in the past decade, such as molecular beam epitaxy, ${ }^{1,2}$ atomic layer deposition, 3,4 pulsed laser deposition, ${ }^{5,6}$ and magnetron sputtering, ${ }^{7}$ have contributed to a giant leap in the synthesis of two-dimensional (2D) materials, as well as the production of technological devices based on these materials. ${ }^{8-11}$ For example, the forefather of $2 \mathrm{D}$ materials, graphene, was obtained using mechanical exfoliation. ${ }^{1,12}$ Another classical 2D material, phosphorene, was fabricated via liquid-phase exfoliation ${ }^{13}$ and pulsed laser exfoliation. ${ }^{5}$ One should not underestimate the contribution of atomistic simulations in the investigations of 2D materials. For instance, first-principles calculations predicted the existence of group $\mathrm{V}$ elements, arsenene and antimonene, ${ }^{14,15}$ for the first time.

The above-mentioned technologies have rendered 2D materials no longer a vision of the future but materials of the present day, and have promoted an extensive study of their structure, properties and applications. However, contemporary knowledge on $2 \mathrm{D}$ materials concludes that each $2 \mathrm{D}$ monolayer possesses some inherent disadvantageous properties. Despite its unique chemical stability, high mechanical durability, and excellent carrier mobility, graphene has no intrinsic band gap. ${ }^{16-20}$ Transition metal dichalcogenides ${ }^{21,22}$ possess sizable and tunable band gaps, but they present several disadvantages, depending on their fabrication methods, such as a relatively low carrier mobility, large volume expansion, and phase conversion. $^{23}$ A recently predicted and isolated group of materials such as phosphorene, arsenene, and antimonene have extraordinary properties, ${ }^{14,24-26}$ including remarkable charge mobility and wide and highly tunable band gaps $(0.3-2 \mathrm{eV})$. However, these materials are chemically active and structurally unstable in standard ambient conditions. ${ }^{27-32}$

As a new class of materials, hybridized composites that combine the advantages and counteract the disadvantages of their constituent compounds are currently emerging in the spotlight. ${ }^{33-38}$ These materials can exist as heterostructures consisting of several layered $2 \mathrm{D}$ materials, ${ }^{33,39-41}$ such as graphene/phosphorene, graphene/indium selenide, boron nitride/phosphorene, or $2 \mathrm{D}$ layers consisting of atoms of different elements. ${ }^{42-44}$ It has been demonstrated that heterostructures formed by $2 \mathrm{D}$ materials, which are unstable in strand ambient conditions, such as phosphorene, and 2D materials, which are stable, such as graphene, are characterized by improved stabilities. ${ }^{37,38,45,46}$ Very recently, a stable

Received: March 3, 2020

Revised: April 12, 2020

Published: April 14, 2020 
allotrope of phosphorus carbide (PC), a 2D monolayer consisting of carbon and phosphorus atoms, has been discovered. $^{47-50}$ It has a wide band gap reaching up to 2.65 $\mathrm{eV}^{48}$ robust superconducting behavior even under tensile strain, ${ }^{51}$ a small effective mass, and extra-high carrier mobility. ${ }^{52}$

It is well-known that the different properties of $2 \mathrm{D}$ materials can be modified by strain engineering. ${ }^{53-57}$ For phosphorene, it has been shown that in-plane strain causes its band gap transition and affects its charge carrier mobility. ${ }^{56,57}$ The strong spatial dependence of the electronic structure upon the rippling of phosphorene along its periodic line profile, which may be used for modulating the injection and confinement of its charge carriers, has been established both theoretically ${ }^{58}$ and experimentally. ${ }^{59}$ However, there have been no similar systematic studies conducted on the rippling of a PC monolayer under a significant out-of-plane deformation.

Graphene and phosphorene nanotubes are currently the subjects of intensive research as base materials for a large variety of technological applications from sensing devices to active optoelectronic elements. ${ }^{60,61}$ For example, phosphorene nanotubes are direct band gap semiconductors and their electronic properties can be modified by strain or electric field engineering. ${ }^{62}$ Meanwhile, graphene nanotubes are common additives to polymer hosts for the manufacturing of advanced composite materials ${ }^{63,64}$ and as electrode materials for batteries and supercapacitors. ${ }^{65}$ Carbon nanotube bundles are promising for shock and vibration protection applications. ${ }^{6-71}$ However, PC nanotubes (PCNTs) are much less studied.

This study systematically investigated the evolution of the atomic structure, the electronic and mechanical properties of a rippled $\alpha$-PC monolayer under large compressive strain. Based on the work, ${ }^{66}$ where layered PC, similar to black phosphorene, was found to be soft when strained along its armchair direction compared with a zigzag direction, our investigation focuses on the armchair rippled $\alpha$-PC monolayer due to its high flexibility. After discovering that the $\alpha$-PC monolayer is able to form nanotubes under large compressive strains applied along its armchair direction, PCNTs are also studied with their thermal stability, atomic structure and electronic and mechanical properties analyzed.

\section{COMPUTATIONAL METHODS}

This work is based on the spin-polarized density-functional theory (DFT) as implemented in the VASP code. ${ }^{72}$ The Perdew-Burke-Ernzerhof (PBE) exchange-correlation functional under the generalized gradient approximation $(\mathrm{GGA})^{73}$ is used for the geometry optimization and electronic structure calculations. Since in the GGA PBE approach the fundamental band gap is usually underestimated, the HSE06 ${ }^{74}$ hybrid exchange-correlation functional is adopted to obtain more accurate band structures. The cutoff energy for plane waves is set at $450 \mathrm{eV}$. The geometries of all structures are fully optimized until the total energy and all forces on atoms converge to less than $10^{-8} \mathrm{eV}$ and $0.01 \mathrm{eV} / \AA$, respectively. The vacuum depth of $15 \AA$ is introduced for all the structures, for both monolayers and nanotubes, to avoid artificial interaction in a supercell. The periodic boundary conditions are applied for the two in-plane transverse directions. The considered supercell of an $\alpha$-PC monolayer is composed of $3 \times 4$ unit cells (12 $\mathrm{C}$ and $12 \mathrm{P}$ atoms). The study of $\alpha$-PC nanotubes (PCNTs) commences with nanotubes of the smallest possible size (4 $\mathrm{C}$ atoms and $4 \mathrm{P}$ atoms). In this work, a PCNT consisting of 40 atoms (PCNT40) is considered, to compromise for the computational demand.

The rippled structures of an $\alpha$-PC monolayer are created by applying a compressive strain along the armchair directions. The compressive strain is defined as

$$
\varepsilon=\frac{l-l_{0}}{l_{0}}
$$

where $l$ and $l_{0}$ are the lattice constants of the strained and initial supercells, respectively. The compression is performed in steps. After each step, the structure is fully relaxed through the technique of energy minimization. Because of the periodic boundary conditions with a fixed period applied laterally, along the armchair direction, the lattice spacing along the zigzag direction remains unchanged.

The Young's modulus of the considered PCNTs is calculated with the aid of the energy-strain relation. ${ }^{75,76} \mathrm{~A}$ set of positive and negative uniaxial strains $\varepsilon$ are applied to the initially unstrained structure along the zigzag direction. For each case considered, the corresponding energy values are calculated. The obtained set of energy values corresponding to each applied strain is then used for a polynomial fitting of energy potential $U(\varepsilon)$. The Young's modulus is computed by

$$
E=\frac{1}{V}\left(\frac{\partial^{2} U(\varepsilon)}{\partial \varepsilon^{2}}\right)_{\varepsilon=0}
$$

where $V$ is the volume of the unit cell of the considered PCNT. Here, it is assumed that $V=2 \pi R l$, where $R$ is the mean radius of the nanotube, while $l$ is the interlayer distance of the $\alpha$-PC monolayer ( $l=4.93 \AA$, as obtained from DFT calculations). As a consequence of the applied uniaxial tensile strain $\varepsilon$, a negative radial strain $\varepsilon_{\mathrm{r}}$ is obtained. The Poisson's ratio $\nu$ is calculated by a linear fitting of $\varepsilon$ versus $\varepsilon_{\mathrm{r}}$ as

$$
v=-\frac{\varepsilon_{\mathrm{r}}}{\varepsilon}
$$

The Young's modulus along the zigzag direction for rippled $\alpha$-PC under the compressive stain applied along the armchair direction is also calculated. It should be noted that internal stresses affect the calculation of the Young's modulus of an elastic body. ${ }^{67}$ However, the elastic modulus of $\alpha$-PC in the armchair direction is 1 order of magnitude smaller than that in the zigzag direction and thus, a compression along the armchair direction produces rather small stresses. Therefore, internal stresses can be neglect when calculating the Young's modulus of $\alpha$-PC along the stiff zigzag direction using the energy-strain relation.

$A b$ initio molecular dynamics (AIMD) calculations ${ }^{77}$ are conducted at $300 \mathrm{~K}$ to verify the thermal stability of PCNTs. The simulation lasts for $10 \mathrm{ps}$ with a time step of $1.0 \mathrm{fs}$ and the temperature is controlled by a Nose-Hoover thermostat. It should be noted that in AIMD calculations the considered unit cell of PCNT40 is repeated three times in the axial direction to show structural evolution of PCNT40 (the total number of atoms is 120) in this direction as well.

\section{RESULTS AND DISCUSSION}

3.1. Electronic Structure and Mechanical Properties of an $\alpha$-PC Monolayer. An $\alpha$-PC monolayer is considered as it was found to be one of the stable allotropes of PC. ${ }^{48,78}$ Moreover, the $\alpha$-PC monolayer was predicted to possess distinguishable features, ${ }^{47-49,52}$ such as a wide band gap and a 
high Young's modulus. The geometry, electronic structure, and Young's modulus of the $\alpha$-PC monolayer are investigated. The considered $\alpha$-PC structure and its unit cell (bounded by the dashed line) are presented in Figure 1a. The obtained relaxed
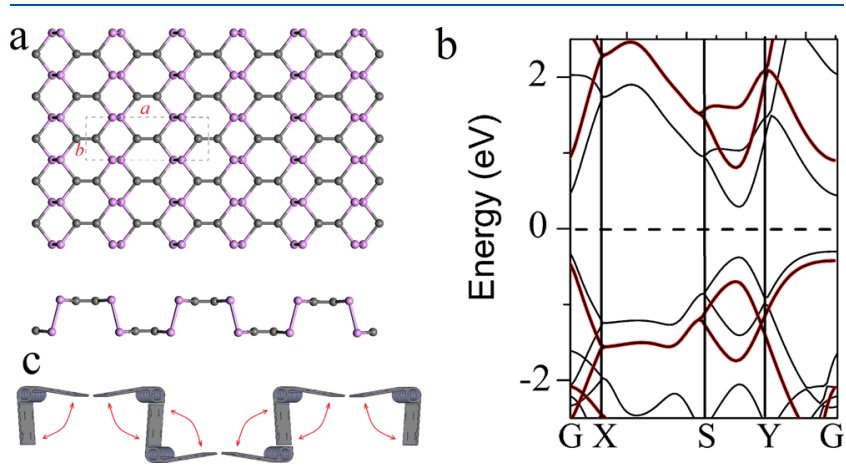

Figure 1. (a) Top and side views of the structure of the $\alpha$-PC monolayer. (b) Band structure of an $\alpha$-PC monolayer. The black and red lines show the band structure calculated by the GGA and HSE methods, respectively. (c) Schematic hingelike structure of an $\alpha$-PC monolayer.

lattice constants $a=8.56 \AA$ and $b=2.92 \AA$ are in good agreement with the previously reported results. ${ }^{47-49}$ Our HSE06 calculations determine the band gap to be $1.32 \mathrm{eV}$. Our PBE GGA calculations predict a qualitatively similar band structure but slightly underestimate the band gap, which is 0.76 $\mathrm{eV}$ in that case. Both values match the reference results obtained by the HSE0 $6^{48}$ and PBE GGA ${ }^{48,49}$ functionals.

To avoid the high computational cost of hybrid functional calculations, we report the results based on the PBE GGA approach. The Young's moduli of the $\alpha$-PC monolayer along its armchair and zigzag directions is found to be 27.08 and $348.69 \mathrm{GPa}$, respectively. The obtained Young's moduli are smaller than those of graphene, ${ }^{79}$ but significantly higher than those of phosphorene. ${ }^{80}$ Interestingly, the $\alpha$-PC monolayer is found to possess a mechanical strength similar to that of a $\gamma$ PC monolayer and superior to that of phosphorene. ${ }^{50}$ The shear modulus of the $\alpha$-PC monolayer reaches up to 81.80 GPa and the Poisson's ratios are 0.25 and 0.019 for tension along the armchair and the zigzag direction, respectively.

3.2. Atomic Structure and Electronic and Mechanical Properties of Rippled $\boldsymbol{\alpha}$-PC. To examine the extreme effects of strain engineering on the rippling of an $\alpha$-PC monolayer and the possibility of formation of nanotubes as a result of compression, compressive strain is applied along its armchair direction (along which the material is more flexible). Figure S1 shows the atomic (upper panel) and band (lower panel) structures of the rippled $\alpha$-PC monolayer under compressive strain ranging from 0 to $48 \%$. The correlation between the band gap size and the applied strain is depicted in Figure 2a. First, for the compressive strain ranging from 0 to $11 \%$, the band gap size slightly decreases from 0.76 to $0.47 \mathrm{eV}$. At the same time, there is no perceptible change to the structure of the rippled $\alpha$-PC monolayer within the range of the applied strains. Further compression (to 16\%) causes a drastic decrease of the band gap size to $0.22 \mathrm{eV}$. Then, for the compressive strain values of 22 and $28 \%$, the band gap size jumps to 0.54 and $0.56 \mathrm{eV}$, respectively, and finally decreases linearly to $0.43 \mathrm{eV}$ as the compressive strain reaches $\sim 48 \%$. It should be noted that an indirect-direct band gap transition occurs in the $\alpha$-PC monolayer when the compressive strain is between 6 and 11\%. Meanwhile, at the compressive strain higher than $11 \%$, a direct band gap is observed and the valence band maximum and conduction bands minimum shift from the $\Gamma$ to the $\mathrm{Y}$ point.

Since rippling usually changes the work function of $2 \mathrm{D}$ materials, ${ }^{81,82}$ the work function of the rippled $\alpha$-PC monolayer is examined. Figure $2 \mathrm{~b}$ shows the work function of the $\alpha$-PC monolayer as a function of the applied compressive strain. The work function of the unstrained monolayered $\alpha$-PC is $4.77 \mathrm{eV}$, which is higher than that of graphene $(4.50 \mathrm{eV})^{83}$ and lower than that of phosphorene $(5.04-5.16 \mathrm{eV}){ }^{84}$ Moreover, the work function of the $\alpha$-PC monolayer is found to have a general tendency to increase with the compressive strain but experience a sharp drop when the strain ranges from 11 to $16 \%$. The observed increase of the work function of $\alpha$-PC may be attributed to the role of the stress accumulated upon the increase of the strain and stretching of the atomic bonds which leads to the shift of the valence band maximum (see Figure S1). ${ }^{85} \mathrm{~A}$ similar linear increase in the work function caused by in-plane strain has recently been reported for $\mathrm{MoS}_{2}$ nanosheets. ${ }^{86}$

According to Figure 2c, the Young's modulus of the rippled $\alpha$-PC monolayer significantly decreases from 348.69 to 290.4 $\mathrm{GPa}$ as the compressive strain increases from 0 to $16 \%$. A drastic plummet of the Young's modulus occurs at the compressive strain of $16 \%$ at the moment of the significant stress accumulation. The subsequent increase of the compressive strain to $32 \%$ induces a rapid increase of the Young's modulus up to $341.43 \mathrm{GPa}$. As the compressive strain increases from 32 to $48 \%$, the Young's modulus slightly decreases to $334.33 \mathrm{GPa}$.

The analysis shows that there is an accumulation of the stress upon the increase of the strain to $\sim 11 \%$, while as the
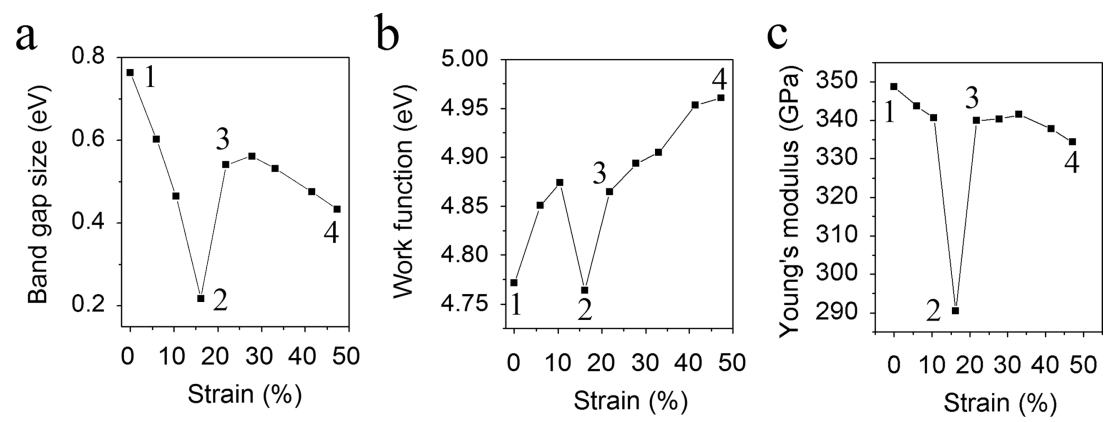

Figure 2. (a) Band gap size, (b) work function, and (c) Young's modulus of a rippled $\alpha$-PC monolayer as a function of compressive strain. The points marked by the numbers 1-4 correspond to the structures shown in Figure 3a. 

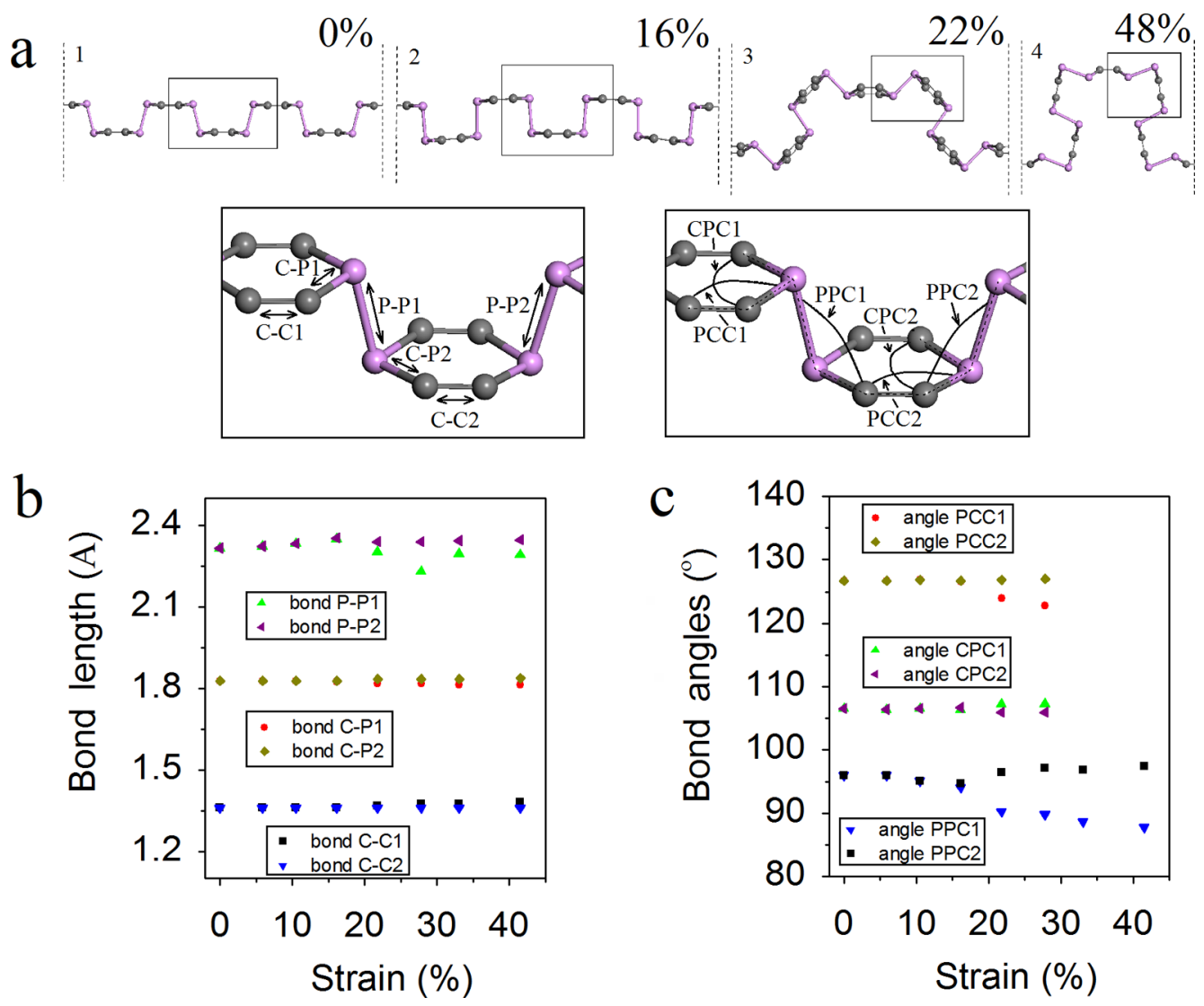

Figure 3. (a) Schematic transformation of $\alpha$-PC under an increasing compressive strain (upper panel). The definition of the structural parameters for $\alpha$-PC (lower panel). The black squares in the upper panel show the area where the structural parameters are measured. The variation of the (b) bond lengths and (c) hinge angles of $\alpha$-PC as a function of the compressive strain.
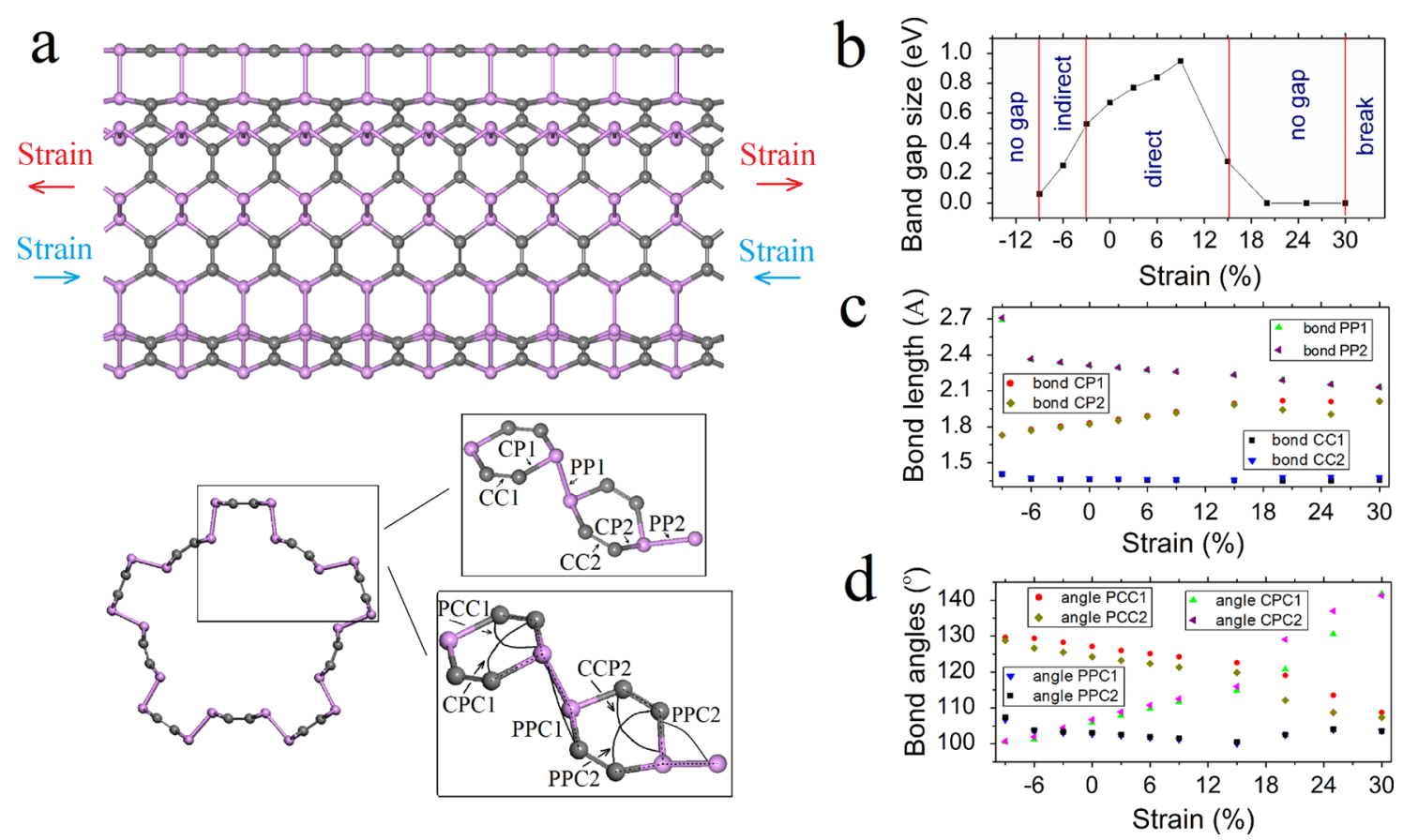

Figure 4. (a) Schematic structure of PCNT40 subjected to strain along its axis (upper panel) and an illustration of the structural parameters of PCNT40 (lower panel). The black squares showing the area where parameters are measured. (b) Band gap size of PCNT40 as a function of strain. Bond (c) lengths and (d) angles of PCNT40 as functions of strain.

compressive stain reaches $\sim 16 \%$, a stress relaxation occurs, which results in a decrease of the band gap size, work function, and Young's modulus. A further increase in the compressive strain, after the release of stress, results in a sharp increase of 

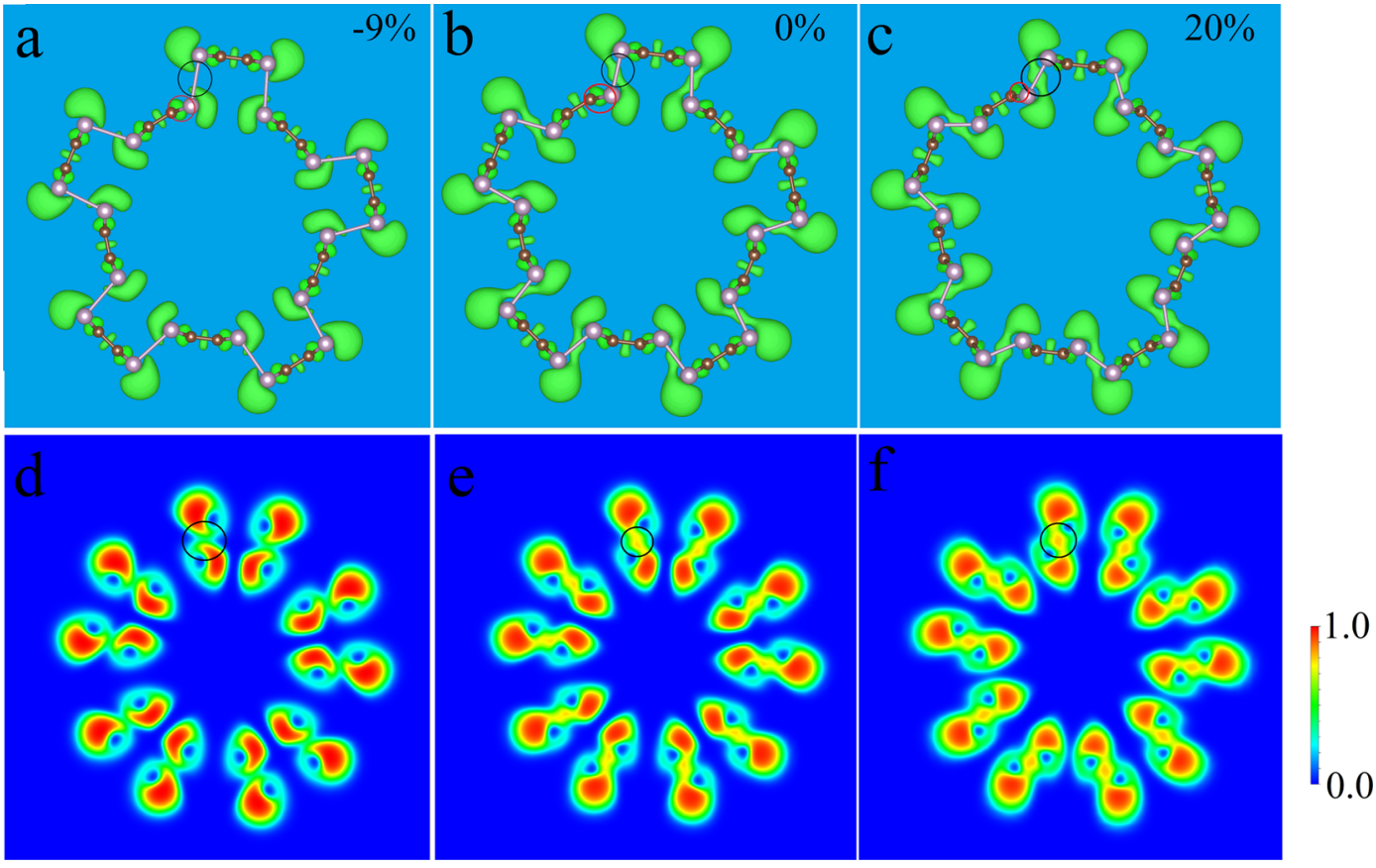

Figure 5. ELFs (upper panel) and ELF maps (lower panel) for PCNT40 (a) under the compressive strain of 9\%, (b) unstrained, and (c) under the tensile strain of $20 \%$. The value for ELFs in $(a)-(c)$ is set to 0.65 .

the band gap size, work function, and Young's modulus of rippled $\alpha$-PC. This can be explained by a specific hinge-like structure of $\alpha$-PC in its armchair direction (Figure 1c, lower panel), which allows $\alpha$-PC to withstand large compressive strains without breaking, similar to phosphorene. ${ }^{56,58}$ To demonstrate this feature, the following structural parameters of the rippled $\alpha$-PC monolayer during the compression deformation are tracked: the bond lengths $\mathrm{P}-\mathrm{P} 1$ and $\mathrm{P}-\mathrm{P} 2$ connecting the hinges, the bond lengths of the hinges $\mathrm{C}-\mathrm{C} 1$, $\mathrm{C}-\mathrm{C} 2, \mathrm{C}-\mathrm{P} 1$, and $\mathrm{C}-\mathrm{P} 2$, and the hinge angles $\mathrm{CPC} 1, \mathrm{CPC} 2$, PCC1, PCC2, PPC1, and PPC2, as shown in the lower panel of Figure $3 \mathrm{a}$. The variations of the bond lengths and angles are presented in Figure $3 b$ and c, respectively.

Indeed, for a compressive strain up to $16 \%$, there are no remarkable changes in the structural parameters except for a slight elongation of the $\mathrm{P}-\mathrm{P} 1$ and $\mathrm{P}-\mathrm{P} 2$ bonds and a slight decrease of the PPC1 and PPC2 angles. Noticeable changes of the structural parameters begin at a compressive strain of $\sim 16 \%$ and beyond. The following changes are observed: (i) oscillation of the $\mathrm{P}-\mathrm{P} 1$ and $\mathrm{P}-\mathrm{P} 2$ bonds and a slight elongation of the $\mathrm{C}-\mathrm{C} 1$ and $\mathrm{C}-\mathrm{C} 2$ bonds (Figure $3 \mathrm{~b}$ ), (ii) a decrease of the PCC1 angle, and (iii) a decrease of the PPC2 angle and an increase of the PPC1 angle (Figure 3c). The observed discrepancies of the angles indicate that the structure folds like a hinge upon reaching a central level of compressive strain $(\sim 16 \%)$, which facilitates the relaxation of the accumulated stress. When the compressive strain exceeds $50 \%$, the breaking of two $\mathrm{P}-\mathrm{P}$ bonds at the base of the structure and the formation of a single $\mathrm{P}-\mathrm{P}$ bond occur with the restructuration of rippled $\alpha$-PC to form a nanotube-like structure. Our AIMD calculations show that transformation of $\alpha$-PC from the rippled structure into the nanotube-like structure under a compressive strain of $\sim 50 \%$ occurs within $\sim 4$ ps (Movie 1 in the Supporting Information (SI)). The nanotube is unstable over a long period of time because in this case, however, from this simulation, the possibility of rolling the rippled $\alpha$-PC to a nanotube is revealed. Previously, nanotubes formed by strain-induced rolling have been predicted experimentally for $\operatorname{In}_{x} \mathrm{Ga}_{1-x}$ As-GaAs membranes. ${ }^{87}$

\subsection{Atomic Structure and Electronic and Mechanical} Properties of $\alpha$-PC Nanotubes. Since the formation of $\alpha$ PC nanotubes by the rippling of an $\alpha$-PC monolayer under large compressive strains is found to be possible, the atomic configurations and the electronic and mechanical properties of these nanotubes are investigated. To determine the smallest size of a stable $\alpha$-PC nanotube (PCNT), AIMD simulations are conducted for PCNTs consisting of 8 (PCNT8), 16 (PCNT16), 24 (PCNT24), 32 (PCNT32), and 40 (PCNT40) atoms. Movie 2 in SI shows the AIMD results for PCNT40, while its atomic structure is shown in Figure 4a. Meanwhile, the atomic structures of PCNT24 and PCNT32 are shown in Figure S2. According to our calculations, PCNT24, PCNT32, and PCNT40 are direct band gap semiconductors with band gap sizes of $0.29,0.57$, and $0.67 \mathrm{eV}$, respectively. The Young's moduli of PCNT24, PCNT32, and PCNT40 along their nanotube axes are $315.87,328.20$, and $333.92 \mathrm{GPa}$. On the other hand, their Poisson's ratios are 0.20, 0.23, and 0.28, respectively. The calculated cohesive energies of PCNT24, PCNT32, and PCNT40 are $-6.695,-6.741$, and $-6.743 \mathrm{eV} /$ atom, respectively, suggesting that the stability of the nanotube slightly increases with its size. Our prediction also suggests that the PCNTs have a Young's modulus about three times less than that of carbon nanotubes ${ }^{88,89}$ but about two times higher than that of phosphorene nanotubes..$^{90,91}$ Meanwhile, the Poisson ratio of the PCNTs is comparable to that of carbon nanotubes. $^{92}$

Figure $4 \mathrm{~b}$ presents the band gap size of PCNT40 as a function of the strain, both compressive and tensile, applied along its nanotube axis (Figure 4a, upper panel). Here, we shall only discuss the results for PCNT40 as PCNT24 and PCNT32 display similar behaviors (Figure S2 and SI for more details). The band gap of PCNT40 decreases rapidly from 0.67 to $0 \mathrm{eV}$ 
as the compressive strain increases from 0 to $9 \%$. Meanwhile, at a compressive strain of $3 \%$, the conduction band minimum shifts from between the $\mathrm{F}$ and $\mathrm{Q}$ points to a $\Gamma$ point (Figure S3), signifying a direct-to-indirect band gap transition. Furthermore, at a compressive strain of $9 \%$ and beyond, the band gap disappears completely, affirming a semiconductor-tometal transition. An increase in the tensile strain from 0 to $9 \%$ leads to an increase of the band gap size from 0.67 to $0.95 \mathrm{eV}$ (Figure 4b). A rapid decrease of the band gap size is observed when the tensile strain is increased from 9 to $20 \%$. Increasing the tensile strain from 20 to $30 \%$ results in the termination of the band gap and a remarkable structure deformation. In particular, a drastic decrease of the PCC1 and PCC2 angles, an increase of the CPC1 and CPC2 angles, as well as a significant elongation of the CP1 and CP2 bonds are observed. Further increasing of a tensile strain would cause nanotube to break.

To understand the changes in the band structure of PCNT40 induced by strain, its structural parameters are analyzed. The following structural parameters (Figure 4a, lower panel) are tracked: (i) the bond lengths of PP1 and PP2 connecting the hinges, (ii) the bond lengths of the hinges $\mathrm{CC} 1$, CC2, CP1, and CP2, and (iii) the hinge angles CPC1, CPC2, PCC1, PCC2, PPC1, and PPC2. The variation of the bond lengths and angles are presented in Figure $4 \mathrm{c}$ and $\mathrm{d}$, respectively. As shown, the band gap size correlates with the bond lengths and angles. Under an increasing compressive strain from 0 to $6 \%$, the band gap size slowly decreases. In addition, the following minor changes under these strains are observed: the bonds PP1 and PP2 are slightly elongated and the bonds $\mathrm{CC} 1$ and $\mathrm{CC} 2$ remain almost unchanged, while the bond angles PCC1, PCC2, PPC1, and PPC2 linearly increase. When the compressive strain is increased from 6 to $9 \%$, the following significant changes in the structural parameters take place: the lengths of the PP1 and PP2 bonds drastically increase and the $\mathrm{CC} 1$ and $\mathrm{CC} 2$ bonds are also elongated noticeably, while the PCC1 angle decreases and the PPC1 and PPC2 angles significantly increase. In addition, there is an almost linear positive correlation between the tensile strain and the band gap size, the bond lengths CP1 and CP2, and the bond angles CPC1 and CPC2. On the other hand, the tensile strain exhibits a negative linear correlation with the bond lengths PP1, PP2, CC1, and CC2 as well as the bond angles PCC1, PCC2, PPC1, and PPC2. It can be concluded that direct-indirect and semiconductor-to-metal transitions in PCNT40 occur mainly due to the transformation of the bond lengths and angles.

To further understand the effects of strain on the electronic structure of PCNT40, the electron localization function (ELF) for PCNT40 under different strains is calculated. For PCNT40 under the compressive strain of $9 \%$ and PCNT40 under the tensile strain of $20 \%$, the isosurface value of 0.65 is adopted in Figure $5 \mathrm{a}-\mathrm{c}$. For the same structures in Figure $5 \mathrm{~d}-\mathrm{f}$, the value of the ELF maps (between 0 and 1) reflects the degree of charge localization in the real space, where 0 represents a free electronic state while 1 represents a perfect localization. As shown in Figure $5 b$ and e, the electrons are packed together at $\mathrm{C}-\mathrm{P}$ bonds (the red circle) and slightly delocalized at $\mathrm{P}-\mathrm{P}$ (the black circle) bonds. As indicated in Figure 5a and d, the compression strain of $9 \%$ leads to an increase of electrons at $\mathrm{P}$ atoms and their localization at $\mathrm{C}-\mathrm{P}$ bonds (the red circle) while at $\mathrm{P}-\mathrm{P}$ bonds (the black circle) electrons are almost delocalized, which suggests the nearly broken $\mathrm{P}-\mathrm{P}$ bonds. Under the tensile strain of $20 \%$ (Figure $5 \mathrm{c}$ and $\mathrm{f}$ ), the electrons at $\mathrm{P}-\mathrm{P}$ bonds exhibit a more highly concentrated distribution while at $\mathrm{C}-\mathrm{P}$ bonds their localization decreases, which indicates a strong covalent chemical bonding between $\mathrm{P}$ atoms.

\section{CONCLUSIONS}

By applying first-principles simulations, we examined and explained the modification of the electronic and mechanical properties of $\alpha$-PC upon rippling under a large compressive strain along the armchair direction. It was found that the Young's modulus of rippled $\alpha$-PC varies from 290.41 to 348.69 GPa under different compressive strains. Rippling also causes an indirect-direct band gap transition in $\alpha$-PC and modifies its work function. In addition, the possibility of restructuring of $\alpha$ $\mathrm{PC}$ into a nanotube-like structure under extreme compressive strains was revealed. $\alpha$-PC nanotubes of different sizes were subsequently studied, and the smallest diameter for an $\alpha$-PC nanotube to be stable at $300 \mathrm{~K}$ were evaluated. The electronic and mechanical properties of stable $\alpha$-PC nanotubes of different diameters were also investigated. The Poisson's ratio of $\alpha$-PC nanotubes was not inferior to that of phosphorene nanotubes, while their Young's modulus was found to exceed that of phosphorene nanotubes. The investigated $\alpha$-PC nanotubes possess a highly tunable band gap under compressive and tensile strains applied along the nanotube axis. Moreover, through strain engineering, it is possible to achieve a semiconductor-to-metal transition in $\alpha$ PC nanotubes or significantly enlarge their band gap size. Therefore, our work suggests rippling as an effective method to tune the mechanical and electronic properties of an $\alpha$-PC monolayer. We also show the possibility of PCNTs fabrication by rippling of a $\alpha$-PC monolayer under extreme compressive strains. Due to their newly found extraordinary properties, PCNTs may eventually replace phosphorene and graphene nanotubes in the design of nanodevices and find application in straintronic, optical, and photovoltaic devices.

\section{ASSOCIATED CONTENT}

\section{Supporting Information}

The Supporting Information is available free of charge at https://pubs.acs.org/doi/10.1021/acs.jpcc.0c01890.

Electronic structure of the $\alpha$-PC monolayer under compressive strains, atomic and electronic structures of PCNT24 and PCNT32, the band structures of PCNT40 under compressive strains (PDF)

AIMD results of transformation of $\alpha$-PC from the rippled structure into the nanotube-like structure (MP4) AIMD results on structural stability of PCNT40 (MP4)

\section{AUTHOR INFORMATION}

\section{Corresponding Author}

Andrey A. Kistanov - Nano and Molecular Systems Research Unit, University of Oulu, Oulu 90014, Finland; 이이.org/ 0000-0001-6175-5296; Email: andrey.kistanov@oulu.fi

\section{Authors}

Stepan A. Shcherbinin - Southern Federal University, Rostovon-Don 344006, Russia

Kun Zhou - School of Mechanical and Aerospace Engineering and Environmental Process Modelling Centre, Nanyang Environment and Water Research Institute, Nanyang Technological University, Singapore 639798; 이이.org/ 0000-0002-2152-8774 
Sergey V. Dmitriev - Institute for Metals Superplasticity Problems, Russian Academy of Science, Ufa 450001, Russia; National Research Tomsk State University, Tomsk 634050, Russia

Elena A. Korznikova - Institute for Metals Superplasticity Problems, Russian Academy of Science, Ufa 450001, Russia

Artur R. Davletshin - Department of Petroleum and Geosystems Engineering, The University of Texas at Austin, Austin, Texas 78712, United States

Complete contact information is available at:

https://pubs.acs.org/10.1021/acs.jpcc.0c01890

\section{Notes}

The authors declare no competing financial interest.

\section{ACKNOWLEDGMENTS}

The authors wish to acknowledge CSC - IT Center for Science, Finland, for computational resources. S.A.S. acknowledges the financial support by the Ministry of Education and Science of the Russian Federation (state task in the field of scientific activity, Southern Federal University), theme N BAS0110/20-3-08IF. K.Z. acknowledges the financial support provided by Nanyang Environment and Water Research Institute (Core Fund), Nanyang Technological University, Singapore. E.A.K. thanks the Russian Foundation for Basic Research, Grant No. 18-32-20158 mol_a_ved. A.A.K. acknowledges the financial support provided $\bar{b}$ by the Academy of Finland (Grant No. 311934).

\section{REFERENCES}

(1) Presel, F.; Tetlow, H.; Bignardi, L.; Lacovig, P.; Tache, C. A.; Lizzit, S.; Kantorovich, L.; Baraldi, A. Graphene growth by molecular beam epitaxy: an interplay between desorption, diffusion and intercalation of elemental C species on islands. Nanoscale 2018, 10, $7396-7406$

(2) Chang, K.; et al. Molecular beam epitaxy growth of superconducting $\mathrm{LiFeAs}$ film on $\mathrm{SrTiO}_{3}(001)$ substrate. Europhys. Lett. 2015, 109, 28003.

(3) Kim, H. G.; Lee, H. B. R. Atomic layer deposition on 2D materials. Chem. Mater. 2017, 29, 3809-3826.

(4) Vervuurt, R. H. J.; Kessels, W. M. M.; Bol, A. A. Atomic layer deposition for graphene device integration. Adv. Mater. Interfaces 2017, 4, 1700232.

(5) Zhang, J.; Shin, H.; Lu, W. Highly ambient-stable few-layer black phosphorene by pulsed laser exfoliation and HEMM. Chem. Commun. 2019, 55, 2601-2604.

(6) Yang, Z.; Hao, J.; Yuan, S.; Lin, S.; Yau, H. M.; Dai, J.; Lau, S. P. Field-effect transistors based on amorphous black phosphorus ultrathin films by pulsed laser deposition. Adv. Mater. 2015, 27, 3748-3754.

(7) Haberkorn, N.; Bengio, S.; Troiani, H.; Suarez, S.; Perez, P. D.; Granell, P.; Golmar, F.; Sirena, M.; Guimpel, J. Thickness dependence of the superconducting properties of $\gamma-\mathrm{Mo}_{2} \mathrm{~N}$ thin films on $\mathrm{Si}(001)$ grown by DC sputtering at room temperature. Mater. Chem. Phys. 2018, 204, 48-57.

(8) Kostarelos, K.; Novoselov, K. S. Graphene devices for life. Nat. Nanotechnol. 2014, 9, 744-745.

(9) Pang, J.; et al. Applications of phosphorene and black phosphorus in energy conversion and storage devices. Adv. Energy Mater. 2018, 8, 1702093.

(10) Schulman, D. S.; Arnold, A. J.; Das, S. Contact engineering for 2D materials and devices. Chem. Soc. Rev. 2018, 47, 3037-3058.

(11) Cheng, J.; Wang, C.; Zou, X.; Liao, L. Recent advances in optoelectronic devices based on $2 \mathrm{D}$ materials and their heterostructures. Adv. Opt. Mater. 2019, 7, 1800441.
(12) Yi, M.; Shen, Z. A review on mechanical exfoliation for the scalable production of graphene. J. Mater. Chem. A 2015, 3, 1170011715.

(13) Yasaei, P.; et al. High-quality black phosphorus atomic layers by liquid-phase exfoliation. Adv. Mater. 2015, 27, 1887-1892.

(14) Zhang, S.; Yan, Z.; Li, Y.; Chen, Z.; Zeng, H. Atomically thin arsenene and antimonene: Semimetal-semiconductor and indirectdirect band-gap transitions. Angew. Chem., Int. Ed. 2015, 54, 31123115.

(15) Akturk, O. U.; Ozcelik, V. O.; Ciraci, S. Single-layer crystalline phases of antimony: Antimonenes. Phys. Rev. B: Condens. Matter Mater. Phys. 2015, 91, 235446.

(16) Oostinga, J. B.; Heersche, H. B.; Liu, X. G.; Morpurgo, A. F.; Vandersypen, L. M. K. Gate-induced insulating state in bilayer graphene devices. Nat. Mater. 2008, 7, 151-157.

(17) Castro, E. V.; Novoselov, K. S.; Morozov, S. V.; Peres, N. M. R.; Lopes dos Santos, J. M. B.; Nilsson, J.; Guinea, F.; Geim, A. K.; Castro Neto, A. H. Biased bilayer graphene: Semiconductor with a gap tunable by the electric field effect. Phys. Rev. Lett. 2007, 99, 216802 .

(18) Zhang, Y. B.; Tan, Y. W.; Stormer, H. L.; Kim, P. Experimental observation of the quantum Hall effect and Berry's phase in graphene. Nature 2005, 438, 201-204.

(19) Lee, J. U.; Yoon, D.; Cheong, H. Estimation of Young's modulus of graphene by Raman spectroscopy. Nano Lett. 2012, 12, 4444-4448.

(20) Wimmer, M.; Adagideli, I.; Berber, S.; Tomanek, D.; Richter, K. Spin currents in rough graphene nanoribbons: Universal fluctuations and spin injection. Phys. Rev. Lett. 2008, 100, 177207.

(21) Radisavljevic, B.; Radenovic, A.; Brivio, J.; Giacometti, V.; Kis, A. Single-layer $\mathrm{MoS}_{2}$ transistors. Nat. Nanotechnol. 2011, 6, 147-150.

(22) Fuhrer, M. S.; Hone, J. Measurement of mobility in dual-gated $\mathrm{MoS}_{2}$ transistors. Nat. Nanotechnol. 2013, 8, 146-147.

(23) Wang, Z.; Mi, B. Environmental applications of 2D molybdenum disulfide $\left(\mathrm{MoS}_{2}\right)$ nanosheets. Environ. Sci. Technol. 2017, 51, 8229-8244.

(24) Pizzi, G.; Gibertini, M.; Dib, E.; Marzari, N.; Iannaccone, G.; Fiori, G. Performance of arsenene and antimonene double-gate MOSFETs from first principles. Nat. Commun. 2016, 7, 12585.

(25) Zhang, S.; Zhou, W.; Ma, Y.; Ji, J.; Cai, B.; Yang, S. A.; Zhu, Z.; Chen, Z.; Zeng, H. Antimonene oxides: Emerging tunable direct bandgap semiconductor and novel topological insulator. Nano Lett. 2017, 17, 3434-3440.

(26) Zhang, S.; Xie, M.; Li, F.; Yan, Z.; Li, Y.; Kan, E.; Liu, W.; Chen, Z.; Zeng, H. Semiconducting group 15 monolayers: A broad range of band gaps and high carrier mobilities. Angew. Chem., Int. Ed. 2016, 55, 1666-1669.

(27) Ares, P.; Aguilar-Galindo, F.; Rodríguez-San-Miguel, D.; Aldave, D. A.; Díaz-Tendero, S.; Alcamí, M.; Martín, F.; GómezHerrero, J.; Zamora, F. Mechanical isolation of highly stable antimonene under ambient conditions. Adv. Mater. 2016, 28, $6332-6336$.

(28) Guo, Y.; Zhou, S.; Bai, Y.; Zhao, J. Oxidation resistance of monolayer group-IV monochalcogenides. ACS Appl. Mater. Interfaces 2017, 9, 12013-12020.

(29) Li, L.; et al. Quantum Hall effect in black phosphorus twodimensional electron system. Nat. Nanotechnol. 2016, 11, 593-597.

(30) Kistanov, A. A.; Cai, Y.; Kripalani, D. R.; Zhou, K.; Dmitriev, S. V.; Zhang, Y. W. A first-principles study on the adsorption of small molecules on antimonene: oxidation tendency and stability. J. Mater. Chem. C 2018, 6, 4308-4317.

(31) Kistanov, A. A.; Cai, Y.; Zhou, K.; Dmitriev, S. V.; Zhang, Y. W. The role of $\mathrm{H}_{2} \mathrm{O}$ and $\mathrm{O}_{2}$ molecules and phosphorus vacancies in the structure instability of phosphorene. 2D Mater. 2017, 4, 015010.

(32) Kistanov, A. A.; Khadiullin, S. Kh.; Zhou, K.; Dmitriev, S. V.; Korznikova, E. A. Environmental stability of bismuthene: oxidation mechanism and structural stability of 2D pnictogens. J. Mater. Chem. C 2019, 7, 9195-9202. 
(33) Cai, Y.; Zhang, G.; Zhang, Y. W. Electronic properties of phosphorene/graphene and phosphorene/hexagonal boron nitride heterostructures. J. Phys. Chem. C 2015, 119, 13929-13936.

(34) Cai, Y.; Pei, Q. X.; Zhang, G.; Zhang, Y. W. Decoupled electron and phonon transports in hexagonal boron nitride-silicene bilayer heterostructure. J. Appl. Phys. 2016, 119, 065102.

(35) Hu, W.; Li, Z.; Yang, J. Structural, electronic, and optical properties of hybrid silicene and graphene nanocomposite. J. Chem. Phys. 2013, 139, 154704.

(36) Wang, H.; Liu, F.; Fu, W.; Fang, Z.; Zhou, W.; Liu, Z. Twodimensional heterostructures: fabrication, characterization, and application. Nanoscale 2014, 6, 12250-12272.

(37) Yuan, J. T.; Najmaei, S.; Zhang, Z. H.; Zhang, J.; Lei, S. D.; Ajayan, P. M.; Yakobson, B. I.; Lou, J. Photoluminescence quenching and charge transfer in artificial heterostacks of monolayer transition metal dichalcogenides and few-layer black phosphorus. ACS Nano 2015, 9, 555-563.

(38) Davletshin, A. R.; Ustiuzhanina, S. V.; Kistanov, A. A.; Saadatmand, D.; Dmitriev, S. V.; Zhou, K.; Korznikova, E. A. Electronic structure of graphene- and $\mathrm{BN}-$ supported phosphorene. Phys. B 2018, 534, 63-67.

(39) Park, S.; Park, C.; Kim, G. Interlayer coupling enhancement in graphene/hexagonal boron nitride heterostructures by intercalated defects or vacancies. J. Chem. Phys. 2014, 140, 134706.

(40) Wang, Z.; Chen, Q.; Wang, J. Electronic structure of twisted bilayers of graphene $/ \mathrm{MoS}_{2}$ and $\mathrm{MoS}_{2} / \mathrm{MoS}_{2}$. J. Phys. Chem. C 2015, 119, 4752-4758.

(41) Kistanov, A. A.; Cai, Y.; Zhou, K.; Dmitriev, S. V.; Zhang, Y. W. Effects of graphene/BN encapsulation, surface functionalization and molecular adsorption on the electronic properties of layered InSe: a first-principles study. Phys. Chem. Chem. Phys. 2018, 20, 1293912947.

(42) Wang, N.; Li, X.; Tu, Z.; Zhao, F.; He, J.; Guan, Z.; Huang, C.; Yi, Y.; Li, Y. Synthesis and electronic structure of boron-graphdiyne with an $s p$-hybridized carbon skeleton and its application in sodium storage. Angew. Chem., Int. Ed. 2018, 57, 3968-3973.

(43) Mortazavi, B.; Shahrokhi, M.; Zhuang, X.; Rabczuk, T. Borongraphdiyne: a superstretchable semiconductor with low thermal conductivity and ultrahigh capacity for $\mathrm{Li}, \mathrm{Na}$ and $\mathrm{Ca}$ ion storage. J. Mater. Chem. A 2018, 6, 11022-11036.

(44) Ullah, S.; Denis, P. A.; Sato, F. Hydrogenation and fluorination of $2 \mathrm{D}$ boron phosphide and boron arsenide: A Density functional theory investigation. ACS Omega 2018, 3, 16416-16423.

(45) Chen, X. L.; et al. Probing the electron states and metalinsulator transition mechanisms in molybdenum disulphide vertical heterostructures. Nat. Commun. 2015, 6, 6088 .

(46) Yuan, Y. J.; et al. The role of bandgap and interface in enhancing photocatalytic $\mathrm{H}_{2}$ generation activity of 2D-2D black phosphorus $/ \mathrm{MoS}_{2}$ photocatalyst. Appl. Catal., B 2019, 242, 1-8.

(47) Huang, X.; et al. Black phosphorus carbide as a tunable anisotropic plasmonic metasurface. ACS Photonics 2018, 5, 31163123.

(48) Guan, J.; Liu, D.; Zhu, Z.; Tomanek, D. Two-dimensional phosphorus carbide: Competition between $\mathrm{sp}^{2}$ and $\mathrm{sp}^{3}$ bonding. Nano Lett. 2016, 16, 3247-3257.

(49) Tan, W. C.; et al. Few-layer black phosphorus carbide fieldeffect transistor via carbon doping. Adv. Mater. 2017, 29, 1700503.

(50) Zhang, W.; Yin, J.; Zhang, P.; Tang, X.; Ding, Y. Twodimensional phosphorus carbide as a promising anode material for lithium-ion batteries. J. Mater. Chem. A 2018, 6, 12029-12037.

(51) Wang, B. T.; Liu, P. F.; Bo, T.; Yin, W.; Eriksson, O.; Zhao, J.; Wang, F. Superconductivity in two-dimensional phosphorus carbide ( $\beta_{0}$-PC). Phys. Chem. Chem. Phys. 2018, 20, 12362-12367.

(52) Wang, G.; Pandey, R.; Karna, S. P. Carbon phosphide monolayers with superior carrier mobility. Nanoscale 2016, 8, $8819-8825$

(53) Bissett, M. A.; Tsuji, M.; Ago, H. Strain engineering the properties of graphene and other two-dimensional crystals. Phys. Chem. Chem. Phys. 2014, 16, 11124-11138.
(54) Wang, V.; Kawazoe, Y.; Geng, W. T. Native point defects in few-layer phosphorene. Phys. Rev. B: Condens. Matter Mater. Phys. 2015, 91, 045433.

(55) Sorkin, V.; Zhang, Y. W. The deformation and failure behaviour of phosphorene nanoribbons under uniaxial tensile strain. 2D Mater. 2015, 2, 035007.

(56) Kou, L.; Ma, Y.; Smith, S. C.; Chen, Ch. Anisotropic ripple deformation in phosphorene. J. Phys. Chem. Lett. 2015, 6, 1509-1513.

(57) Peng, X.; Wei, Q.; Copple, A. Strain-engineered direct-indirect band gap transition and its mechanism in two-dimensional phosphorene. Phys. Rev. B: Condens. Matter Mater. Phys. 2014, 90, 085402 .

(58) Kistanov, A. A.; Cai, Y.; Zhou, K.; Dmitriev, S. V.; Zhang, Y. W. Large electronic anisotropy and enhanced chemical activity of highly rippled phosphorene. J. Phys. Chem. C 2016, 120, 6876-6884.

(59) Quereda, J.; et al. Strong modulation of optical properties in black phosphorus through strain-engineered rippling. Nano Lett. 2016, 16, 2931-2937.

(60) Ling, X.; Wang, H.; Huang, S.; Xia, F.; Dresselhaus, M. S. The renaissance of black phosphorus. Proc. Natl. Acad. Sci. U. S. A. 2015, $112,4523-4530$.

(61) Liu, B.; Zhou, K. Recent progress on graphene-analogous 2D nanomaterials: properties, modeling and applications. Prog. Mater. Sci. 2019, 100, 99-169.

(62) Guo, H.; Lu, N.; Dai, J.; Wu, X.; Zeng, X. C. Phosphorene nanoribbons, phosphorus nanotubes, and van der Waals multilayers. J. Phys. Chem. C 2014, 118, 14051-14059.

(63) Dimiev, A. M.; Lu, W.; Zeller, K.; Crowgey, B.; Kempel, L. C.; Tour, J. M. Low-loss, high-permittivity composites made from graphene nanoribbons. ACS Appl. Mater. Interfaces 2011, 3, 46574661.

(64) Dimiev, A. M.; Zakhidov, D.; Genorio, B.; Oladimeji, K.; Crowgey, B.; Rothwell, E. J.; Kempel, L. C.; Tour, J. M. Permittivity of dielectric composite materials comprising graphene nanoribbons. The effect of nanostructure. ACS Appl. Mater. Interfaces 2013, 5, $7567-7573$

(65) Li, L.; Raji, A. R. O.; Tour, J. M. Graphene-wrapped $\mathrm{MnO}_{2}$ graphene nanoribbons as anode materials for high-performance lithium ion batteries. Adv. Mater. 2013, 25, 6298-6302.

(66) Liu, H.; Neal, A. T.; Zhu, Z.; Luo, Z.; Xu, X.; Tomanek, D.; Ye, P. D. Phosphorene: An unexplored $2 \mathrm{D}$ semiconductor with a high hole mobility. ACS Nano 2014, 8, 4033-4041.

(67) Cao, A. Y.; Dickrell, P. L.; Sawyer, W. G.; Ghasemi-Nejhad, M. N.; Ajayan, P. M. Super-compressible foamlike carbon nanotube films. Science 2005, 310, 1307-1310.

(68) Korznikova, E. A.; Rysaeva, L.Kh.; Savin, A. V.; Soboleva, E. G.; Ekomasov, E. G.; Ilgamov, M. A.; Dmitriev, S. V. Chain model for carbon nanotube bundle under plane strain conditions. Materials 2019, 12, 3951.

(69) Rysaeva, L.Kh.; Korznikova, E. A.; Murzaev, R. T.; Abdullina, D. U.; Kudreyko, A. A.; Baimova, J. A.; Lisovenko, D. S.; Dmitriev, S. V. Elastic damper based on the carbon nanotube bundle. Facta Universitatis Series: Mechanical Engineering 2020, 18, 1-12.

(70) Abdullina, D. U.; Korznikova, E. A.; Dubinko, V. I.; Laptev, D. V.; Kudreyko, A. A.; Soboleva, E. G.; Dmitriev, S. V.; Zhou, K. Mechanical response of carbon nanotube bundle to lateral compression. Computation 2020, 8, 27.

(71) Savin, A. V.; Korznikova, E. A.; Dmitriev, S. V.; Soboleva, E. G. Graphene nanoribbon winding around carbon nanotube. Comput. Mater. Sci. 2017, 135, 99-108.

(72) Kresse, G.; Furthmüller, J. Efficient iterative schemes for ab initio total-energy calculations using a plane-wave basis set. Phys. Rev. B: Condens. Matter Mater. Phys. 1996, 54, 11169.

(73) Perdew, J. P.; Burke, K.; Ernzerhof, M. Generalized gradient approximation made simple. Phys. Rev. Lett. 1996, 77, 3865.

(74) Heyd, J.; Scuseria, G. E.; Ernzerhof, M. Hybrid functionals based on a screened Coulomb potential. J. Chem. Phys. 2003, 118, 8207. 
(75) Le Page, Y.; Saxe, P. Symmetry-general least-squares extraction of elastic data for strained materials from $a b$ initio calculations of stress. Phys. Rev. B: Condens. Matter Mater. Phys. 2001, 63, 174103.

(76) Domínguez-Rodríguez, G.; Tapia, A.; Aviles, F. An assessment of finite element analysis to predict the elastic modulus and Poisson's ratio of singlewall carbon nanotubes. Comput. Mater. Sci. 2014, 82, 257-263.

(77) Henkelman, G.; Uberuaga, B. P.; Jonsson, H. A climbing image nudged elastic band method for finding saddle points and minimum energy paths. J. Chem. Phys. 2000, 113, 9901.

(78) Li, F.; Liu, X.; Wang, J.; Zhang, X.; Yang, B.; Qu, Y.; Zhao, M. A promising alkali-metal ion battery anode material: $2 \mathrm{D}$ metallic phosphorus carbide $\left(\beta_{0}\right.$-PC). Electrochim. Acta 2017, 258, 582-590.

(79) Lee, C.; Wei, X.; Kysar, J. W.; Hone, J. Measurement of the elastic properties and intrinsic strength of monolayer graphene. Science 2008, 321, 385-388.

(80) Elahi, M.; Khaliji, K.; Tabatabaei, S. M.; Pourfath, M.; Asgari, R. Modulation of electronic and mechanical properties of phosphorene through strain. Phys. Rev. B: Condens. Matter Mater. Phys. 2015, 91, 115412.

(81) Cui, T. T.; Li, J. C.; Gao, W.; Jiang, Q. Geometric and electronic structure of multilayered graphene: synergy of the nondirective ripples and the number of layers. Phys. Chem. Chem. Phys. 2018, 20, 2230-2237.

(82) Sorkin, V.; Zhang, Y. W. The structure and elastic properties of phosphorene edges. Nanotechnology 2018, 29, 235707.

(83) Lee, E. J. H.; Balasubramanian, K.; Weitz, R. T.; Burghard, M.; Kern, K. Contact and edge effects in graphene devices. Nat. Nanotechnol. 2008, 3, 486-490.

(84) Cai, Y.; Zhang, G.; Zhang, Y. W. Layer-dependent band alignment and work function of few-layer phosphorene. Sci. Rep. 2015, 4, 6677.

(85) Lanzillo, N. A.; Simbeck, A. J.; Nayak, S. J. Strain engineering the work function in monolayer metal dichalcogenides. J. Phys.: Condens. Matter 2015, 27, 175501.

(86) Shakya, J.; Kumar, S.; Kanjilal, D.; Mohanty, T. Work function modulation of molybdenum disulfide nanosheets by introducing systematic lattice strain. Sci. Rep. 2017, 7, 9576.

(87) Chun, I. S.; Challa, A.; Derickson, B.; Hsia, K. J.; Li, X. Geometry effect on the strain-induced self-rolling of semiconductor membranes. Nano Lett. 2010, 10, 3927-3932.

(88) Kim, H.; Wang, M.; Lee, S. K.; Kang, J.; Nam, J. D.; Ci, L.; Suhr, J. Tensile properties of millimeter-long multi-walled carbon nanotubes. Sci. Rep. 2017, 7, 9512.

(89) Yu, M. F.; Lourie, O.; Dyer, M. J.; Moloni, K.; Kelly, T. F.; Ruoff, R. S. Strength and breaking mechanism of multiwalled carbon nanotubes under tensile load. Science 2000, 287, 637-640.

(90) Sorkin, V.; Zhang, Y. W. Mechanical properties of phosphorene nanotubes: a density functional tight-binding study. Nanotechnology 2016, 27, 395701.

(91) Zhang, W.; Yin, J.; Zhang, P.; Ding, Y. Strain/stress engineering on the mechanical and electronic properties of phosphorene nanosheets and nanotubes. RSC Adv. 2017, 7, 51466-51474.

(92) Yakobson, B. I.; Avouris, P. Mechanical properties of carbon nanotubes. In Carbon Nanotubes; Dresselhaus, M. S., Dresselhaus, G., Avouris, P., Eds.; Topics in Applied Physics; Springer; Berlin, Heidelberg, 2001; Vol. 80. 